\title{
ESTUDO DE UM ANALISADOR DE MOBILIDADE ELÉTRICA DE NANOPARTÍCULAS ATRAVÉS DE AEROSSÓIS POLIDISPERSOS
}

\author{
I. B. C. COLICHIO, E. C. M. CAMARGO, F. O. AROUCA, J. J. R. DAMASCENO \\ Universidade Federal de Uberlândia, Faculdade de Engenharia Química \\ E-mail para contato: icherulli@gmail.com
}

\begin{abstract}
RESUMO - A nanotecnologia possui uma vasta gama de aplicações, alguns exemplos são as indústrias de eletrônicos, farmacêutica e de filtros de ar. Para produção e controle de qualidade de partículas tão pequenas foi desenvolvido o analisador de mobilidade elétrica de nanopartículas (Nano-DMA). O Nano-DMA foi projetado e construído na FEQUI/UFU para estudo da difusão de partículas nanométricas de um aerossol polidisperso, a fim de classificá-las de acordo com sua distribuição de tamanho, produzindo uma corrente monodispersa que apresenta nanopartículas que atendem aos parâmetros de diâmetro desejáveis. O design desse equipamento consiste em dois eletrodos cilíndricos concêntricos. É aplicada uma tensão no cilindro interno, enquanto o cilindro externo é aterrado, estabelecendo um campo elétrico entre eles. Realizando a passagem do aerossol polidisperso por esse campo é possível determinar o número e o diâmetro das partículas que atravessam o equipamento e saem no aerossol monodisperso. Neste trabalho, foi avaliado o desempenho do Nano-DMA utilizando-se duas amostras de aerossol de $\mathrm{NaCl}$ em diferentes concentrações. Os resultados obtidos mostraram que a concentração de sal presente na corrente de entrada influencia na concentração de nanopartículas do aerossol monodisperso produzido, ou seja, quanto maior a concentração de $\mathrm{NaCl}$ utilizada, maior será a concentração de partículas no aerossol de saída.
\end{abstract}

\section{INTRODUÇÃO}

Segundo o documento PAS71 comissionado pelo Departamento Inglês de Comércio e Indústria (DTI), pode ser considerada uma nanopartícula um corpo que tenha dimensão na ordem de $100 \mathrm{~nm}$ ou menor. Por serem tão pequenas, apresentam características que as diferenciam de seu material de origem. Materiais compostos de partículas nanométricas possuem significativas propriedades mecânicas, óticas e magnéticas que são desejáveis em muitas aplicações na engenharia e indústria modernas.

De acordo com Chen et al. (1997), o domínio das partículas ultrafinas na indústria de discos rígidos para computador, por exemplo, é primordial, pois a distância entre a cabeça de leitura eletromagnética e os discos em que os dados são gravados (platters) está se aproximando de zero com o objetivo de armazenar maior quantidade de informações em uma área menor do disco. Para que isso seja possível, é fundamental a produção de nanopartículas de alta qualidade. Isso se torna viável através do controle de qualidade dessa produção, realizando análises de tamanho, composição, estrutura e morfologia dessas nanopartículas. 
As mesmas características que tornam as nanopartículas tão notáveis podem também representar efeitos nocivos à saúde e ao meio ambiente. Essas partículas são liberadas inadvertidamente no ar e, segundo estudos epidemiológicos realizados por Oberdorster et al. (2004) e Pope (1996), podem causar problemas respiratórios, doenças cardiovasculares e, ainda, penetrar no cérebro causando doenças neurológicas.

A síntese de nano-aerossóis (gases com nanopartículas sólidas suspensas) é fundamental para estudo da questão ambiental e de saúde pública, e crescimento e desenvolvimento da área de nanotecnologia. Numa vasta gama de processos de obtenção de material particulado ultrafino, um importante parâmetro de análise é o tamanho da partícula, e um classificador otimizado para a faixa de variação de diâmetro desejado (nano) como pode-se observar nos trabalhos de Chen et al. (1997), é fundamental. Dentre todos os instrumentos de classificação de aerossóis, o Nano-DMA se mostra eficiente, oferecendo controle de qualidade das partículas em tempo real.

O objetivo deste projeto é produzir nanopartículas monodispersas em correntes gasosas utilizando-se um equipamento projetado e construído na FEQUI/UFU com o intuito de promover a separação de nanopartículas em aerossóis polidispersos, analisando a interferência da concentração de $\mathrm{NaCl}$ nos aerossóis de saída.

\section{TEORIA}

\subsection{Funcionamento do Nano-DMA}

O Nano-DMA é composto de dois eletrodos cilíndricos concêntricos dispostos verticalmente. Dois fluxos entram na região de classificação do Nano-DMA: um contendo o aerossol polidisperso que apresenta partículas com variadas mobilidades elétricas, e outro ar invólucro isento de partículas. O primeiro é aplicado na região periférica do anel, e o segundo, próximo ao cilindro interno. Aplica-se uma certa tensão no eletrodo de menor diâmetro, enquanto o eletrodo com maior raio é aterrado, criando um campo elétrico radial. As partículas do aerossol polidisperso são, então, arrastadas pelo ar de entrada para a parte inferior da região de classificação e sofrem a ação do campo elétrico presente entre os eletrodos, como mostrado na Figura 1.

Partículas carregadas positivamente são atraídas pelo eletrodo central que se mantém a uma tensão negativa, e as negativas são repelidas. Se a partícula apresenta alta mobilidade elétrica, ela ficará retida em uma região superior do Nano-DMA, enquanto as de baixa mobilidade elétrica (e maiores diâmetros) atravessam mais facilmente para a região inferior. Portanto, as partículas com determinado diâmetro (função da tensão aplicada) que atravessam com sucesso a região de classificação do Nano-DMA são coletadas numa corrente de saída, dando origem ao aerossol monodisperso que contém partículas em uma certa faixa de mobilidade elétrica. Partículas maiores do que o limite superior da faixa desejada constituirão a corrente de excesso. 
Figura 1 - Representação do Nano-DMA e trajetória da partícula do aerossol

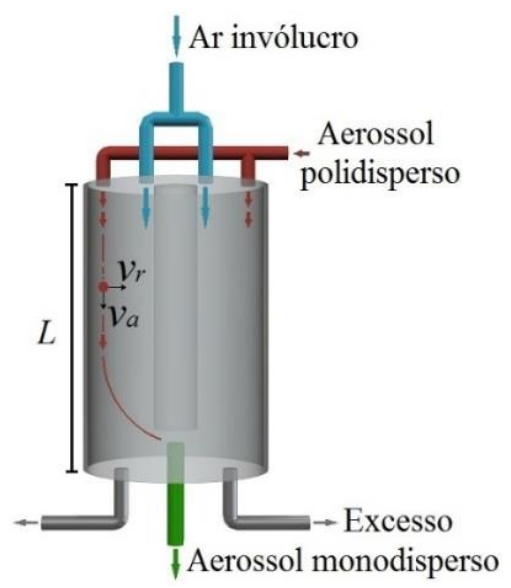

\subsection{Cálculo do diâmetro das partículas do aerossol monodisperso}

Adotando $Q$ como a vazão volumétrica na entrada, $E$ o campo elétrico, $Z_{e}$ a mobilidade elétrica e $r_{1}$ e $r_{2}$ os raios dos cilindros interno e externo respectivamente, considera-se que toda partícula que entra no Nano-DMA possui uma velocidade axial $v_{a} \mathrm{e}$ uma velocidade radial $v_{r}$, dadas pelas Equações 1 e 2:

$$
\begin{aligned}
& v_{a}=\frac{Q}{\pi\left(r_{2}^{2}-r_{1}^{2}\right)} \\
& v_{r}=E Z_{e}
\end{aligned}
$$

Admite-se que as partículas que chegam à corrente de saída contendo aerossol monodisperso devem percorrer a distância entre os dois eletrodos no mesmo período de tempo que leva para percorrer a distância vertical $L$. Ao aplicar esse conceito nas Equações 1 e 2, e integrando-se a Equação 2, é possível obter uma equação para cálculo da mobilidade elétrica em função da tensão aplicada $(V)$, representada pela Equação 3.

$$
Z_{e}=\frac{Q \ln \left(\frac{r_{2}}{r_{2}}\right)}{2 V L \pi}
$$

Pode-se também definir a mobilidade elétrica como sendo a velocidade da partícula $(v)$ por unidade de força do campo aplicado $(E)$, como mostrado na Equação 4:

$$
Z_{e}=\frac{v}{E}
$$

Tomando a força eletrostática $F_{e}$ sobre a partícula e a força de arraste $F_{d}$, definidas pelas Equações 5 e 6, respectivamente, obtém-se, por substituição na Equação 4, a mobilidade elétrica da partícula, dada pela Equação 7.

$$
F_{e}=n e E
$$




$$
\begin{aligned}
F_{d} & =\frac{3 \pi \mu D_{p} v}{C} \\
Z_{e} & =\frac{C n e}{3 \pi \mu D_{p}}
\end{aligned}
$$

A Equação 7 fornece a mobilidade elétrica da partícula de diâmetro $D_{p}$ que possui $n$ cargas, sendo $e$ a carga elementar $\left(1,6 \times 10^{-19}\right.$ Coulomb), $C$ o fator de escorregamento de Cunningham e $\mu$ a viscosidade do gás.

Igualando a Equação 3 e a Equação 7, é possível determinar a Equação 8 que fornece uma expressão para cálculo do diâmetro das partículas:

$$
D_{p}=\frac{2 n e C L V}{3 Q \mu \ln \left(\frac{r_{2}}{r_{1}}\right)}
$$

\section{MATERIAIS}

- Rotâmetros: para controle das vazões de escoamento do gás;

- Mini compressor de ar: produz incremento na vazão e pressão nas correntes de entrada;

- Compressor de ar: dilui as partículas dos testes experimentais;

- Atomizador comercial: gera aerossol polidisperso uniforme;

- Filtros de ar de alta eficiência: realiza alta purificação do ar;

- Secador de difusão: através da sílica gel remove o excesso de umidade do aerossol polidisperso;

- Fonte neutralizadora de raio-X: produz íons de ar positivos e negativos;

- Fonte de alta tensão: introduz tensão ajustável no Nano-DMA;

- Nano-DMA: equipamento projetado e construído pela FEQUI-UFU que seleciona partículas produzindo uma corrente monodispersa;

- Contador de Partículas (CPC): classifica partículas e mede suas concentrações.

\section{PROCEDIMENTO EXPERIMENTAL}

Duas soluções com volume de aproximadamente $80 \mathrm{~mL}$ preparadas com $\mathrm{NaCl}$ e água ultrapura foram utilizadas, uma com concentração de $0,1 \mathrm{~g} / \mathrm{L}$ de $\mathrm{NaCl}$ e outra de $0,5 \mathrm{~g} / \mathrm{L}$. Primeiramente, o ar advindo do compressor passava pelos filtros de alta eficiência para remoção de impurezas e era conduzido ao reservatório do atomizador comercial onde estava a solução de $\mathrm{NaCl}$. Aqui, era gerado o aerossol polidisperso que seguia para o secador de difusão, este era formado por dois cilindros concêntricos com o espaçamento entre eles preenchido por sílica-gel com intuito de minimizar a umidade do aerossol de entrada. Posteriormente, o aerossol adentrava o neutralizador de raio-X, produzindo uma corrente de aerossol neutralizado para classificação. 
Essa corrente, então, entrava pelo topo do Nano-DMA, que, conectado à fonte de alta tensão, produzia a corrente de saída. Foram aplicadas 22 medidas de tensões entre 20 - 4000V em cada uma das duas soluções. O contador de partículas realizava, então, as medidas de concentração das correntes de entrada, saída e excesso. Para maior precisão de resultados foram realizadas tréplicas do experimento.

\section{RESULTADOS E DISCUSSÃO}

A seleção da faixa de diâmetro desejada é função da tensão aplicada no eletrodo central, que determina a força do campo elétrico produzido. Portanto, ajustando-se valores de tensões pode-se regular a dimensão das partículas coletadas.

Conforme apresentado por Dalcin (2013), pela distribuição de cargas proposta por Wiedensohler e pela da Equação 7, pode-se concluir que quanto maior a partícula, menor será sua mobilidade elétrica, todavia, quanto maior sua carga, maior será sua capacidade de se movimentar através do campo aplicado.

Nas Figuras 2 e 3 estão representados o comportamento obtido para o aerossol monodisperso e de excesso para as concentrações de $\mathrm{NaCl}$ de $0,1 \mathrm{~g} / \mathrm{L}$ e de $0,5 \mathrm{~g} / \mathrm{L}$, após a realização das tréplicas para cada concentração, constando o desvio padrão obtido para cada $D_{p}$. Essas figuras foram geradas pelo software Origin a partir dos dados coletados pelo contador de partículas.

Figura 2 - Composição de concentrações do aerossol monodisperso
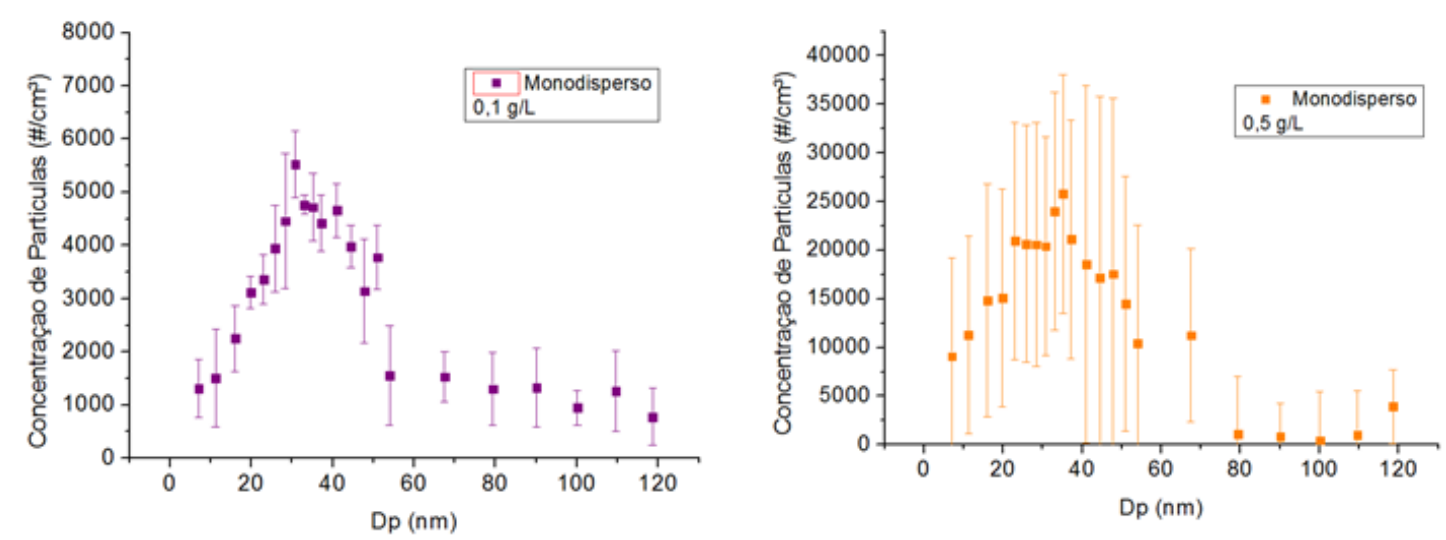

Figura 3 - Composição de concentrações na corrente de excesso
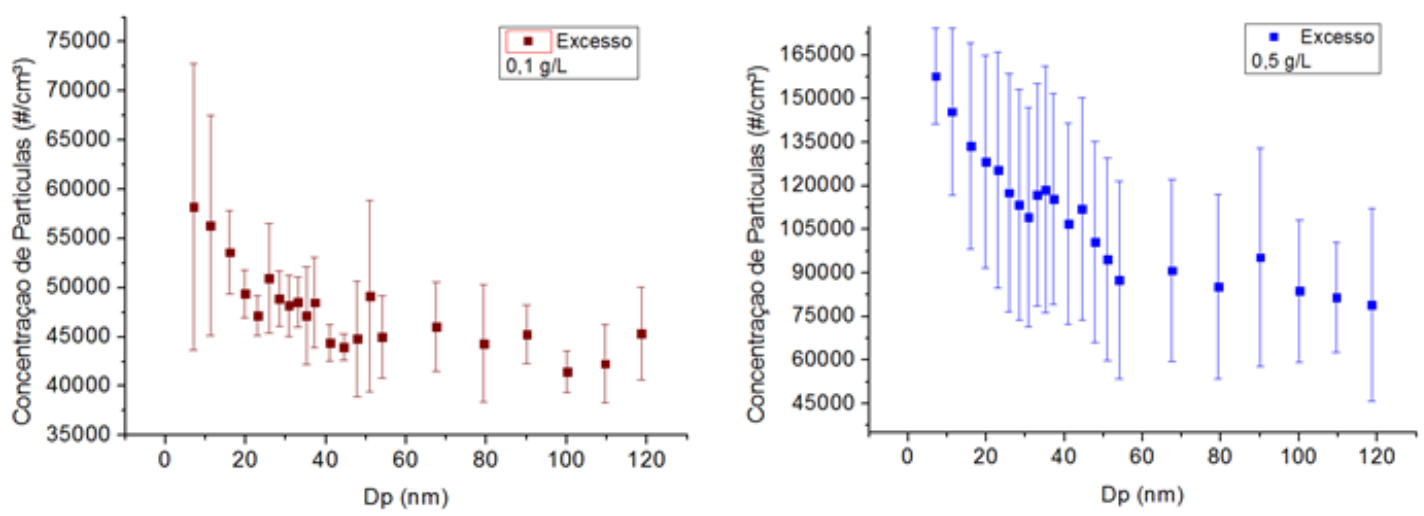
Como pode ser observado pela análise das Figuras 2 e 3 , a concentração de nanopartículas presentes nos aerossóis de saída aumenta quanto maior for a concentração de $\mathrm{NaCl}$ utilizado. Observa-se que para um mesmo $D_{p}$, há uma maior concentração de partículas na solução de $0,5 \mathrm{~g} / \mathrm{L} \mathrm{NaCl}$. Os resultados obtidos a partir das duas amostras indicam a capacidade de classificação do equipamento quando se trata de partículas nanométricas, sendo que isto pode ser afirmado pela análise do exposto por Camargo (2015). Testes subsequentes mostram-se necessários, mas os resultados apresentados por esse equipamento foram bastante promissores.

\section{CONCLUSÕES}

Nos testes realizados com o Nano-DMA foi possível produzir nanopartículas monodispersas em correntes gasosas, além de se observar que a concentração de partículas aumenta com o aumento da concentração salina utilizada.

\section{AGRADECIMENTOS}

Agradecemos à Fundação de Amparo à Pesquisa do Estado de Minas Gerais (FAPEMIG) pelos recursos concedidos para a elaboração do projeto.

\section{REFERÊNCIAS}

BRITISH STANDARDS INSTITUTION (BSI). PAS 71 - Vocabulary for nanoparticles, 2011.

CAMARGO, E.C.M. Estudo do Desempenho de um Analisador de Mobilidade Elétrica de Nanopartículas. Universidade Federal de Uberlândia, 2013, Brasil. Dissertação de Mestrado.

CHEN, D. R.; PUI, D. Y. H.; HUMMES, D.; FISSAN, H.; QUANT, F. R.; SEM, G. J. Design and evaluation of a nanometer aerosol differential mobility analyzer (nano-DMA). $J$. Aerosol Sci., v. 29, p. 497-509, 1998.

DALCIN, M.G. Geração de nanopartículas monodispersas em correntes gasosas. Universidade Federal de Uberlândia, 2013, Brasil. Tese de Doutorado.

OBERDÖRSTER, G.; SHARP, Z.; ATUDOREI, V.; ELDER, A.; GELEIN, R.; KREYLING, W.; COX, C. Translocation of inhaled ultrafine particles to the brain. Inhalation toxicology, v. 16, p. 437-445, 2004.

POPE, C. A. Particulate pollution and health: a review of the Utah valley experience. $J$. of exposure analysis and environmental epidemiology, v. 6, p. 23-34, 1995. 